\section{A New Relativity Theory of the Unified} Physical Field.

I HAVE succeeded in applying to the above question what appears to be an important new conception regarding the significance of relativity mathematics with noteworthy results.

I have abandoned altogether the quasi-geometrical interpretation with such hazy notions as parallel displacement in a curved space of $n$ dimensions. Instead, we construct an indeterminate vector field by means of Eddington's displacement rule, considered now as an association rule, and identify it at every point with the velocity a material particle might have if present there and then. Vector lines in this field are necessarily the orbits of material particles under any physical conditions.

We now construct an indeterminate tensor field, and by its means define the invariant magnitude of the elementary arc of these vector lines.

Actual physical vector and tensor fields are necessarily determinate at every point, and these are defined as usual by applying the association rule round a closed loop. This gives the usual symmetric and antisymmetric field tensors.

When the antisymmetric field tensor vanishes the vector lines reduce to Einstein's so-called geodesics. The electric field is given by the antisymmetric tensor, and here the vector lines become mathematically equivalent to the known orbit equations. Maxwell's first set of field laws are identically satisfied, and the set referring to electric charge and current are approximately true for small fields. In strong fields in the neighbourhood of atomic nuclei the electron orbits reduce again to geodesics, independent of negligible radiation due to their acceleration, and Maxwell's second set of laws no longer hold. This is obviously very strong support for our theory. But further: the introduction of field laws which reduce to Einstein's in the pure gravitation field appear to lead to a principle of selection among the orbits in nuclear regions.

Before leaving England this summer I prepared a hurried account of the above theory intended for publication in the Proceedings of the Royal Society. It unfortunately gave no explanation of the mathematics, which latter were faulty at one point. A careful and complete revision has now proved the soundness of the theory, and publication will follow as quickly as possible.

Physics Department,

Yenching University,

Peking, Nov. 26, 1929.

\section{Aucuba or Yellow Mosaic of the Tomato Plant : Reaction of Infected Juice.}

IN the course of investigations into the physiology of virus diseases, it was observed that if the expressed juice from a tomato plant infected with aucuba mosaic disease be mixed with an aqueous colloidal solution of tomato chlorophyll, that is, the mixed green pigments extracted from fresh plant material by the method of Willstätter and Stoll from a healthy tomato plant, a marked action occurs under suitable conditions. The most striking manifestation of this action is the development of a brown colour and, apparently, the destruction of a greater or lesser amount of the chlorophyll. This browning action is either not shown or is shown to a very much smaller degree by the sap from a healthy plant.

The reaction appears to show a marked sensitiveness to light and to be greatly accelerated by a light intensity of the order of 800 foot candles at a temperature of about $30^{\circ} \mathrm{C}$., thus showing a certain parallel to the development of symptoms of mosaic disease in the living plant. The reaction has not been found to occur to the same degree with a sample of infected sap which has been boiled for a few seconds, neither does it appear to be directly correlated with the oxidase or peroxidase content of the sap.

Under the conditions of experiment, the browning has not been observed to take place to any marked degree with infected sap exposed to light in the absence of colloidal chlorophyll, although it is a matter of general observation that the sap from an infected plant either is brown or becomes brown on keeping. Yet at the dilutions employed in the experiments referred to, this brown colour is scarcely perceptible and cannot account for the marked change on exposure to light, which is not shown by the sap in the absence of colloidal chlorophyll.

These observations suggest that this reaction may provide a quick and quantitative means of studying in vitro the nature of the virus and its reactions, the examination of which up to the present has entailed prolonged inoculation experiments.

Critical investigations are in hand with the view of obtaining a fuller understanding of this and cognate phenomena.

W. F. BeWLey.

Experimental and Research Station, Bernard J. Bolas. Cheshunt, Herts.

\section{The Vapour Density of Sodium.}

THERE is plenty of evidence in the literature to the effect that the vapour of sodium is monatomic. Robitsch (Ann. Physik, 38, 1027; 1912) determined the velocity of sound in the vapour at the boiling point and calculated the ratio of specific heats to be 1.68 , and Taylor (Phys. Rev., 28, 576; 1926) found no evidence of molecules at pressures of $10^{-2} \mathrm{~mm}$. Other evidence, which need not be considered here, tends to corroborate this opinion.

With the discovery of the band spectra of diatomic molecules in the vapour of the alkali metals, it became necessary to consider the possibility of molecular association in the vapour state. Carelli and Pringsheim (Zeit. f. Physik, 44, 643; 1927) calculated the heat of dissociation for the potassium molecule to be 0.61 volt. A thermodynamic calculation indicates that potassium vapour will be associated to a very slight extent. For sodium the best value of the heat of dissociation is that of Loomis (Phys. Rev., 31, 323; $1928), 1 \cdot 0 \pm 0 \cdot 1$ volt, and thermodynamic calculations have been made which predict that there will be more molecules than atoms in the vapour of sodium near the boiling point. Recently, Mr. E. G. Walters, working with me in this laboratory, has re-determined very carefully the vapour pressure and vapour density of sodium. A complete account of the work will be published elsewhere, but it may be noted here that an apparent molecular weight of 25 was found for the saturated vapour at $706^{\circ} \mathrm{C}$. By substituting the value of the equilibrium constant calculated from this result in the equation of Gibson and Heitler (Zeit. f. Physik, 49,$465 ; 1928$ ) a value of about 0.75 volt is obtained for the heat of dissociation. The agreement between this result and the value obtained by Loomis from band spectra cannot be considered entirely satis factory without extending unduly the limits of probable error for one or both of the determinations.

There is no reason to change the value for the entropy of monatomic sodium vapour calculated by Rodebush (Proc. Nat. Acad., 13, 185 ; 1927).

University of Illinois,

W. H. Rodebush. Nov. 25, 1929.

No. 3143 , VoL. 125] 